\title{
Integrierte Kommunikation - theoretisches Konstrukt oder praktisches Handlungskonzept
}

\author{
Markus DROWATZKY, PhD \\ Hochschule Mittweida, University of Applied Sciences \\ E-mail: markus.drowatzky@hs-mittweida.de
}

Zusammenfassung. Steigender Konkurrenzdruck und eine sich verschärfende Wettbewerbssituation erhöhen den Druck auf Unternehmen und Organisationen. Längst reichen deshalb klassische Werbeformen und ein Denken in klassischen Marketingdimensionen nicht mehr aus. Kommunikation ist längst keine alleinige Aufgabe der Presse- und Kommunikationsstäbe mehr, sondern hat sich zu einer umfassenden und alle Bereiche betreffenden Komplexaufgabe gewandelt. Als Lösung für die neuen und vielfältigen Herausforderungen hat sich in den vergangenen zwanzig Fahren das Konzept der integrierten Kommunikation herausgestellt. Die Integrierte Kommunikation ist dabei sowohl als ein strategischer und operativer Prozess zu verstehen, der zum Ziel hat, sowohl verschiedene externe und interne Kommunikatoren einzubeziehen, als auch eine einheitliche Botschaft an die verschiedenen und diversen Zielgruppen zu senden, um ein konsistentes Erscheinungsbild zu vermitteln. Der Beitrag diskutiert die Relevanz der integrierten Kommunikation für Unternehmen und Organisationen beispielhaft an einer Auswahl existierender Modelle. Im Zentrum steht dabei die Frage nach der Praxisrelevanz, Anwendung und Umsetzbarkeit der theoretisch-modellhaften Beschreibung im realen Umfeld. Durch die Untersuchung ausgewählter Modelle werden die verschiedenen Herangehensweisen dargestellt, sowie Merkmale betrachtet, 
die als Erfolgsfaktoren für das Gelingen der integrierten Kommunikation in der Praxis dienen können. Dabei besteht die größte Herausforderung darin, dass die Modelle auch die Komplexität der heutigen Unternehmensund Organisationsrealität abbilden.

Schlüsselwörter: Kommunikation; Integrierte Kommunikation; Unternehmenskommunikation; Modelle; Theorie.

\title{
Integrated Communication - Theoretical Construct or Practical Approach
}

\begin{abstract}
More and more competitors and an intensifying competitive situation increase the pressure on companies and organizations. Traditional forms of advertising and old ways of the communication thinking are no longer sufficient. Communication has long been a comprehensive task affecting all areas. Over the past twenty years, the concept of integrated communication has emerged as the solution to the new and diverse challenges. Integrated communication is a strategic and operational process with the aim of involving various external and internal communicators, as well as sending a uniform message to the various target groups in order to convey a consistent image. The article discusses the relevance of integrated communication for companies and organizations using a selection of existing models as examples. The focus is on the question of practical relevance, application and feasibility of the theoretical models in a real environment. By examining selected models, the different approaches are presented and characteristics are considered that can serve as success factors for the success of integrated communication in practice. The greatest challenge is that the models also reflect the complexity of today's corporate and organizational reality.
\end{abstract}

Steigender Konkurrenzdruck und eine verschärfte Wettbewerbssituation erhöhen den Druck auf Organisationen und Unternehmen. Längst sind klassische Werbeformen und ein Denken in klassischen Marketingdimensionen Auslaufmodelle zur Selbstdarstellung und Leistungsschau. Die interne Kommunikation hat zudem deutlich an Wichtigkeit gewonnen, um die eigenen Mitarbeiter zu motivieren, sie zu Aushängeschildern des eigenen Unternehmens zu machen und die eigene Unternehmenskultur $\mathrm{zu}$ verstetigen. Unternehmen sind heute sogenannte „brands“ (Marken) geworden, deren Werte und Leistungen kommuniziert werden müssen (Kirchner 2001, 17). Die Unternehmenskommunikation ist deshalb darauf ausgerichtet, möglichst viele Kanäle zu bespielen. Dabei gilt es, Inhalte und Prozesse 
zu bündeln, Verantwortlichkeiten festzulegen und grundsätzliche inhaltliche und formale Kriterien für die Kommunikation zu etablieren. Diese Anforderungen verlangen nach einem Konzept, das möglichst viele Anforderungen bündelt - ein Versprechen, dass das Konzept der integrierten Kommunikation erfüllen möchte.

\section{Integrierte Kommunikation - eine Definition}

Das Konzept der Integrierten Kommunikation basiert auf einem holistischen Ansatz der versucht, mit einer interdisziplinären Lösung die Unterteilung beispielsweise in die Disziplinen der Werbung, des Sponsorings, der Public Relations, der internen Kommunikation zu überwinden. Laut Schultz geht es um den Perspektivwechsel hin zu einer Kommunikation, so wie sie die Kunden in ihrem Alltag wahrnehmen (Schultz et al., 1993, xvii). Das begriffliche Konstrukt wird von vielen Forschern unterschiedlich wahrgenommen. Einige Wissenschaftler bezeichnen die Integrierte Kommunikation als gedankliches Konstrukt (vgl. Peppels 1994, 449), andere als Prozess oder Optimierungskonzept. Entsprechend der Ansätze existieren eine Vielzahl von Definitionen, die jeweils eine andere Wahrnehmung auf einzelne Elemente oder die Zielgruppen aufweisen (vgl. Pelsmacker et al., 2001; Eagle, Kitchen, Bulmer, 2007; Kerr et al., 2008, 514).

In den letzten Jahren und insbesondere im deutschsprachigen Raum haben sich Bruhn und Zerfaß mit ihren Definitionen durchgesetzt. Zerfaß beschreibt die Integrierte Kommunikation als Prozess und Ergebnis der Abstimmung und Koordination aller Kommunikationsaktivitäten, die übergreifende Zusammenarbeit aller zuständigen Mitarbeiter mit einer gemeinsamen Strategie sowie unternehmensweit abgestimmte Ziele und Erfolgsindikatoren auf verschiedenen Wirkungsebenen (vgl. Zerfaß, 2017, 10). Diese Definition greift die relevanten Felder der integrierten Kommunikation auf, ohne dabei zu sehr ins Detail zu gehen. Zerfaß selbst nutzt für seine Definition als Basis die Definition von Manfred Bruhn (vgl. Zerfaß, 2017, 10). Aufgrund der Ausführlichkeit und der allgemeinen Akzeptanz in Deutschland, bildet die Definition von Bruhn die Grundlage für die weiteren Betrachtungen.

„Integrierte Kommunikation ist ein strategischer und operativer Prozess der Analyse, Planung, Organisation, Durchführung und Kontrolle, der darauf ausgerichtet ist, aus den differenzierten Quellen der internen und externen Kommunikation von Unternehmen eine Einheit herzustellen, um ein für die Zielgruppen der Kommunikation konsistentes Erscheinungsbild des Unternehmens bzw. eines Bezugsobjektes der Kommunikation zu vermitteln." (Bruhn, 2009, 22).

In der Wissenschaft wird das Konzept der integrierten Kommunikation insbesondere in den USA seit Mitte der 90er Jahre diskutiert (vgl. Kirchner, 2001, 
19) - Bruhn setzt den Beginn der Diskussion sogar noch zwei Jahrzehnte eher an (vgl. Bruhn, 2009, 1). Unter dem Begriff „Integrated Marketing Communications“ (IMC) (vgl. Kirchner, 2001, 19) gab es einen umfassenden Diskurs der bis heute nicht gänzlich abgeschlossen ist. Kirchner bilanzierte zu Beginn der 2000er Jahre, dass das Konzept noch immer in der Entwicklungsphase stecke (vgl. Kirchner, 2001, 19).

Zentrale Frage bleibt daher, die Praxisrelevanz des Konzepts. Im Zentrum steht die Frage, ob die integrierte Kommunikation nur den Status einer wissenschaftlichen Theorie innehat und die modellhafte Abstraktionsebene nie verlässt oder doch einen so großen Praxisbezug aufweist, dass daraus Handlungsempfehlungen für Unternehmen generiert werden können.

Der Begriff der Theorie ist in diesem Fall die Summe jenes gesicherten Wissens, das aus dem „Zusammenwirken von Erfahrung und Denken [...]“ entsteht (Seiffert \& Radnitzky, 1994, 368) bzw. Theorien sind Systeme, die thematisch und logisch miteinander verknüpfte Gesetzesaussagen über (Kausal-)Zusammenhänge abbilden (vgl. Burkart, 1998, 405). Da es sich in der wissenschaftlichen Betrachtung der integrierten Kommunikation um die modellhafte Beschreibung handelt, ist vorab klar, dass es sich bei den Modellen um keine exakten Abbildungen der Realität, sondern graphische Vereinfachungen realer Erscheinungsformen und Prozesse handelt (vgl. Maletzke, 1998, 56).

Für die Annäherung an die zentrale Frage ist neben der wissenschaftlichen Beschreibung der integrierten Kommunikation, die offensichtlich bereits vorliegt, die Praxisrelevanz entscheidend. Deshalb soll zunächst der Bedarf an einem Modell der integrierten Kommunikation anhand des Bedarfs von Unternehmen und deren alltäglichen Kommunikationsherausforderungen geklärt werden.

\section{Notwendigkeit der Integrierten Kommunikation}

Der grundlegende Ansatz der Kommunikation eines Unternehmens liegt darin, ein möglichst widerspruchsfreies, glaubhaftes und klares Bild gegenüber unterschiedlichen (Teil-)Öffentlichkeiten und Zielgruppen zu vermitteln, dass sowohl leicht wiedererkannt und gelernt wird und sich im besten Fall an den individuellen Kommunikationsbedürfnissen orientiert (vgl. Kirchner, 2001, 33; Bruhn, 2009, 1). Erschwert wird dieses Ziel durch die Informationsüberlastung der Zielgruppen, die bereits Mitte der 80er Jahre nach einer Studie von KroeberRiel (1987) bei 98 Prozent (vgl. Kroeber-Riel \& Esch, 2004, 16; vgl. Brünne, Esch, Ruge, 1987) lag. Eine Wiederholung der Studie zu Beginn der 2000er kam zu dem Schluss, dass höchstens fünf Prozent der gesendeten Unternehmensinformationen überhaupt die Chance haben, von der Zielgruppe wahrgenommen, geschweige denn verarbeitet zu werden (vgl. Kroeber-Riel \& Esch, 2004, 17). Durch die stetige Entwicklung der elektronischen Medien und deren Bedeutungsgewinn, ist die Informationsüberlastung noch einmal größer. Zusätzlich sieht Schultz einen Verlust 
der Kommunikationskontrolle, der insbesondere durch die Entwicklung der neuen Medien entstanden ist (Schultz et al., 1993, 38).

In der Folge liegen viele Informationen beim Rezipienten nur in Fragmenten vor. Wenn diese Fragmente nicht klar zuzuordnen, beziehungsweise konsistent und verständlich vorliegen, werden sie ignoriert (vgl. Kirchner, 2001, 33; Merten, 1994, 50ff). Es besteht die Notwendigkeit, die Kommunikationsbemühungen stärker zu konzentrieren und intern zu einer besseren Orientierung und extern zu einer größeren Erfolgsrate zu führen (Bruhn, 2009, 4; Kirchner, 2001, 33f). Können Fragmente eindeutig einem Unternehmen zugeordnet werden, ergibt sich ein konsistentes Bild. Dies ist das Ziel der integrierten Kommunikation, die gleichzeitig eine bessere Ausnutzung der Potentiale und Synergien der Kommunikationsaktivitäten und im besten Fall eine Kostenersparnis bei Erhöhung der Wirksamkeit verspricht (vgl. Kirchner, 2001, 33f).

Die Notwendigkeit in der Praxis durch die geänderten Rahmen- und Marktbedingungen in einem sich stetig ändernden Umfeld sind damit gegeben. Die integrierte Kommunikation scheint ein Konzept zur Lösung der beschriebenen Herausforderungen zu sein. Doch es gibt kein zentral etabliertes Konzept der integrierten Kommunikation, welches unmittelbar als Lösung taugt. Vielmehr haben sich in den vergangenen Jahrzehnten verschiedene Ansätze herauskristallisiert, die sich durch ihre Kriterien und zentralen Ansätze unterscheiden.

Eine aktuelle Betrachtung der integrierten Kommunikation aus den USA nach den zentralen Arbeiten von Don E. Schultz und Mitautoren verfolgt den Ansatz, dem Wirkungsverlust des klassischen Marketings die umfassende Integration aller instrumentellen Kommunikationsaktivitäten entgegenzusetzen ist (vgl. Schultz, Schultz, 2004). In Europa wird diese Betrachtung insbesondere von Philip J. Kitchen und Patrick de Pelsmacker vertreten (Kitchen \& Pelsmacker, 2004).

In den letzten Jahren haben sich in Deutschland insbesondere Manfred Bruhn, Ansgar Zerfaß und Franz Rudolf Esch mit dem Konzept der integrierten Kommunikation beschäftigt (Bruhn, 1992, 2014; Bruhn et al., 2016; Esch, 1998, 2011; Zerfaß,1996,2017). Sie verbindet mit dem amerikanischen Ansatz, dass die Integration vorrangig durch eine Abstimmung der Kommunikationsmaßnahmen umzusetzen und an den Unternehmenszielen auszurichten ist. Diese Herangehensweise an das Thema Integration hat sich als äußerst produktiv erwiesen (vgl. Zerfaß, 2017, 9) und zahlreiche wissenschaftliche Arbeiten inspiriert. Der Vorteil in dieser Betrachtung liegt in der konkreten Aufarbeitung und Bestimmung von Werkzeugen für die Planung und Kontrolle und in der Vielzahl an empirischen Studien zum Status Quo und der Entwicklungsperspektive für Unternehmen, die in die Betrachtungen eingeflossen sind.

Ein weiterer Ansatz ist, nicht die Kommunikation in den Fokus zu stellen, sondern Ziele und Strategien des Unternehmens in den Mittelpunkt zu rücken (Steinmann \& Zerfaß, 1995; Zerfaß, 1996, 2010; Zerfaß \& Piwinger, 2014). 
Die Verschiedenartigkeit der Ansätze und Modelle führt in der Wissenschaft zu verschiedenen Klassifizierungen. Stumpf unterscheidet drei grundlegende Ansätze (vgl. Stumpf, 2005, 19f):

1. Managementorientierte Ansätze (vgl. Moore \& Thorson 1996; Bruhn, 1992),

2. stakeholderorientierte Ansätze (vgl. Gronstedt, 1996; Zerfaß, 1996),

3. gestaltungs- und wirkungsorientierte Ansätze (vgl. Kroeber-Riel, 1993; Esch, 2001).

Innerhalb der managementorientierten Ansätze gilt dieintegrierteKommunikation als mehrstufiger Prozess, der vom Unternehmen gezielt zu planen und Stufe für Stufe umzusetzen ist. Stakeholderorientierte Ansätze berücksichtigen die Integration sämtlicher Bezugsgruppen des Unternehmens und stellen hierfür einen konzeptionellen Rahmen bereit. Die gestaltungs- und wirkungsorientierten Ansätze legen ihren Fokus auf die Wirkung des Zusammenspiels formaler und inhaltlicher Kriterien vor allem im Bereich extern orientierter Kommunikationsinstrumente wie der Werbung. Die Aufgliederung der Modelle ist nicht absolut trennscharf, da beispielsweise auch die managementorientierten Ansätze eine gewisse Stakeholder-Orientierung aufweisen können. Da der letzte Ansatz der Gestaltungsund Wirkungsorientierung für sich genommen kein eigenes Modell ist, sondern dessen Wirkung und einzelne Elemente untersucht und fragmentiert in den anderen Ansätzen aufgeht, soll dieser Ansatz in der folgenden Betrachtung nicht weiterverfolgt werden. Um ein möglichst breites Bild der Ansätze zu vermitteln, werden nachfolgend die managementorientierten Modelle von Thorson \& Moore und Bruhn, sowie die stakeholderorientierten von Gronstedt und Zerfaß vorgestellt.

\section{Modell nach Thorson und Moore}

Das Modell nach Thorson und Moore begreift die Integrierte Kommunikation als strategische Koordination verschiedener Kommunikationskanäle. Das Ziel ist die Optimierung der Ansprache von Stakeholdern (Konsumenten, Handel, Fachpublikum, ...) durch die Koordination und Abstimmung der Elemente des Marketingmixes, wie Werbung, Öffentlichkeitsarbeit, Verkaufsförderung, Direktmarketing und Verpackungsdesign (vgl. Thorson, Moore, 1996, 1). Die Autoren haben mit ihrem Konzept drei zentrale Anliegen:

1. Kombination verschiedener Kommunikationsinstrumente, wie Radio, Fernsehen oder Zeitungen in der Öffentlichkeitsarbeit und in der Mediawerbung zur Erzielung maximaler Mediawerbung.

2. Integrierte Kommunikationskampagnen, die ohne Werbung als Leitinstrument auskommen.

3. Integrierte Kommunikation, die nicht nur ausschließlich auf den Konsumenten ausgerichtet ist (vgl. Thorson \& Moore, 1996, 2ff). 
Das Modell der integrierten Kommunikation weist eine starke Ausrichtung auf den Konsumenten auf und besteht aus drei zentralen Merkmalen. Basis bildet der Markenkern, der sowohl für die Gegenwart und auch für die Vorhaben in der Zukunft untersucht werden muss. Anschließend folgt eine Eruierung und Definition des Zielmarktes, gleichzeitig muss die Zielgruppe identifiziert werden. Die Prüfung beinhaltet das Abfragen der Erwartungshaltung der Kunden und die Einflüsse auf den Kunden. Im Ergebnis werden die passenden Instrumente bestimmt, die aus den Bereichen Public Relations, Direct Marketing, Promotion, Verpackung und Werbung kommen.

Thorson und Moore entwickeln aufbauend auf den Merkmalen einen vierstufigen Managementprozess (Thorson \& Moore, 1996, 142ff). Nach der Identifizierung der Zielgruppen rund um Kunden und beeinflussende Faktoren (Handel, Medien) erfolgt eine Marktsegmentierung mit der Analyse, wie ein Absatz des Produkts oder der Dienstleistung kommunikativ begleitet werden kann. Im dritten Schritt werden die Erkenntnisse genutzt, um Kommunikationsbotschaften festzulegen und an den richtigen Stellen zu platzieren. Abschließend werden die Budgets für die einzelnen Ziele, Segmente und Kommunikationsmaßnahmen verteilt.

\section{Modell nach Manfred Bruhn}

Manfred Bruhns Modell wird den managementorientierten Ansätzen zugeordnet. Er gilt aufgrund seiner Vielzahl an Publikationen und seiner seit 1991 währenden Auseinandersetzung als wichtigster deutscher Vertreter im Bereich der integrierten Kommunikation.

Bruhn sieht in der Praxis ein sehr diffuses Begriffsverständnis. Er beschreibt die Integrierte Kommunikation als: (...) ein strategischer und operativer Prozess der Analyse, Planung, Organisation, Durchführung und Kontrolle, der darauf ausgerichtet ist, aus den differenzierten Quellen der internen und externen Kommunikation von Unternehmen eine Einheit herzustellen, um ein für die Zielgruppen der Kommunikation konsistentes Erscheinungsbild des Unternehmens bzw. eines Bezugsobjektes der Kommunikation zu vermitteln.“ (Bruhn, 2009, 22). Zentrales Kriterium der Definition ist die Schaffung einer Einheit, in der sich die gesamten Kommunikationsaktivitäten eines Unternehmens integrieren. Daneben muss die Kommunikation so positioniert sein, dass sie die strategische Positionierung des Unternehmens im Kommunikationswettbewerb unterstützt.

Die Integrierte Kommunikation unterscheidet sich trotz vieler Gemeinsamkeiten von dem Konzept der Corporate Identity. Sie betrachtet auch die Marken des Unternehmens als wichtigstes kommunikatives Bezugsobjekt während die Corporate Identity vor allem die Vereinigung zwischen Selbstbild und Fremdbild des Unternehmens beinhaltet, was den Blickpunkt ausschließlich auf das Unternehmen lenkt. Prinzipiell steht für Bruhn die Integrierte Kommunikation in Abhängigkeit 
von der Markenstrategie, die er als vorgelagerte strategische Marketingentscheidung sieht und an der sich das Bezugsobjekt der integrierten Kommunikation - das Unternehmen als Ganzes oder die Marke/Marken des Unternehmens - auszurichten hat. Im Ergebnis muss die inhaltliche, formale und zeitliche Integration hinsichtlich des Bezugsobjektes bei den Zielgruppen der Kommunikation ein einheitliches Erscheinungsbild hervorrufen.

Manfred Bruhn klassifiziert drei Formen der Integration. Die inhaltliche Integration hat das Ziel, die Kommunikationsinstrumente und -mittel thematisch aufeinander abzustimmen. Dadurch wird das Kommunikationsziel des einheitlichen Erscheinungsbildes erreicht. Dies gelingt beispielsweise durch einheitliche Slogans, Kernbotschaften, Kernargumente, Schlüsselbilder, Verbindungen visueller Bilder mit akustischen Signalen usw (Bruhn, 2009, 80f).

Dieformale Integration der Kommunikation bezieht sich aufdie Vereinheitlichung der Gestaltungsprinzipien für die Kommunikationsinstrumente und -mittel mit dem Ziel einer einheitlichen Form des Erscheinungsbildes. Als Gestaltungsprinzipien kommen beispielsweise die Verwendung einheitlicher Unternehmens- sowie Markenzeichen, Logos nach vorgegebenen formalen Richtlinien (insb. Schrifttyp, Größe, Farbe oder Bilder) in Frage. Diese Gestaltungsprinzipien haben auch Gültigkeit für die Integration auf horizontaler als auch auf vertikaler Ebene.

Idealtypische Elemente der formalen Integration können beispielsweise sein:

- Markenname

- Logo

- Slogan

- Typografie

- Layout

- Farben

- Bilder

Die formale Integration unterstützt die inhaltliche Integration bei der Verbindung der Positionierungsinhalte mit einer Marke bzw. dem Bezugsobjekt der Kommunikation. Die Marke lässt sich über visuelle Erkennungsmerkmale leichter im Gedächtnis der Zielgruppe verankern. (Bruhn, 2009, 84ff).

Die zeitliche Integration umfasst die Abstimmung des Einsatzes von Kommunikationsinstrumenten und - mitteln aufeinander. Damit einher geht der Wunsch eines einheitlichen Erscheinungsbildes. Die zeitliche Integration umfasst zwei Teilaspekte:

1. Die zeitliche Abstimmung zwischen verschiedenen Kommunikationsinstrumenten, damit sich alle eingesetzten Kommunikationsinstrumente gegenseitig im zeitlichen Einsatz unterstützen.

2. Die zeitliche Kontinuität eines Kommunikationsinstruments, um Wirkungsverlusten durch häufigen Wechsel entgegenzuwirken und beim Rezipienten Wiederholungsund Lerneffekte zu erzielen. 
Bruhn selbst schätzt ein, dass die inhaltliche Integration am schwierigsten umzusetzen ist, da die Variablen nur wenig kontrollierbar sind und die Inhalte mittel- bis langfristigen Zielen und Botschaften der Kommunikation dienen. Allerdings lässt sich durch die inhaltliche Konsistenz die größte Wirkung erzielen. Dem gegenüber lässt sich die formale Integration leichter und schneller aber weniger effektiv umsetzen (Bruhn, 2009, 88f).

Für Unternehmen läuft der Prozess für die Integration nach einem Plan mit neun verschiedenen Punkten ab. Zunächst beginnen sie mit der Analyse der Kommunikationsinstrumente, -mittel und der grundlegenden Kommunikationssituation. Unter Einbeziehung der Marktforschung und deren Instrumenten werden die internen und externen Einflussvariablen und deren gegenseitige Beeinflussung erhoben. Variablen können dabei marktbezogene, kundenbezogene, handelsbezogene, wettbewerbsbezogene, umfeldbezogene und umweltbezogene Einflussvariablen sein. Die Analysemethoden schwanken je nach Einsatzzweck, denkbar wären beispielsweise eine SWOT-Analyse zur allgemeinen Strukturierung der Situationsvariablen sowie spezielle Analysen, wie die Lebenszyklusanalyse oder die Imageanalyse (Bruhn, 2009, 146ff).

Im zweiten Schritt werden die Ziele der integrierten Kommunikation festgelegt. Dabei ist es elementar, die Ziele so genau wie möglich festzulegen. Nur so können exakte Schlussfolgerungen für die Kommunikationsaktivitäten getroffen werden und es lässt sich ein hoher Grad an Verbindlichkeit für die Kontrolle der Kommunikationsaktivitäten erzielen. Der managementorientierte Ansatz von Bruhn orientiert sich dabei auch an den Unternehmenszielen, die als Vorlage für die Kommunikationsziele dienen. Auch für sie gilt eine Verbindlichkeit und Operationalisierbarkeit. Die Ziele werden schwerpunktmäßig für alle Zielgruppen bestimmt, um eine einheitliche Darstellung zu erreichen. In dieser Stufe werden auch die Ziele für die einzelnen Kommunikations-instrumente festgelegt. Die Festlegung der Zielgruppen erfolgt in der darauffolgenden Stufe. Nach Bruhn können Zielgruppen beispielsweise Kunden, Handel, Mitarbeiter und Öffentlichkeit sein. In der Praxis werden die Zielgruppen jedoch weiter unterteilt, um die passenden und effizienten Kommunikationsinstrumente und -mittel zuordnen zu können.

In Stufe vier werden die Instrumente der integrierten Kommunikation ausgewählt und kategorisiert. Die Instrumente werden dabei hinsichtlich ihrer Eignung zur Erreichung des Ziels und in ihrer Beziehung zur Zielgruppe analysiert.

Die Eignung zur Zielerreichung kann mit Hilfe einer Zielerreichungsmatrix und der Vergabe von entsprechenden Punktbewertungen gemessen wird. Die Beziehung zwischen Zielgruppe und Instrument kann durch eine hierarchische Kategorisierung der Kommunikationsinstrumente abgelesen werden. Die Instrumente unterteilt Bruhn dabei in vier Kategorien (Bruhn \& Boenigk, 1999, 70ff) mit dem Ziel einer Ordnung der Instrumente innerhalb der integrierten Kommunikation:

1. Leitinstrumente: welche andere Instrumente stark beeinflussen, aber selbst weniger beeinflusst werden (bspw. klassische Werbung). 
2. Integrationsinstrumente: welche andere Instrumente eher weniger beeinflussen noch selbst beeinflusst werden (bspw. Event-Marketing oder Sponsoring).

3. Kristallisationinstrumente: die sowohl andere Instrumente stark beeinflussen, als auch selbst stark beeinflusst werden (bspw. Öffentlichkeitsarbeit, Mitarbeiterkommunikation oder Verkaufsförderung).

4. Folgeinstrumente: die ausschließlich von anderen Instrumenten beeinflusst werden (bspw. Messen und Ausstellungen).

Schließlich werden in Schritt fünf die Planungselemente in einem strategischen Konzept zusammengeführt. Bruhn bezeichnet diesen Schritt deshalb als Kernstück des integrierten Planungsprozesses und besteht deshalb aus den voneinander abhängigen Kernelementen der strategischen Positionierung des Unternehmens, der Definition der kommunikativen Leitidee und der Spezifizierung der Leitinstrumente. Ziel ist Erstellung eines Sollbilds unter Einbeziehung der Hauptziele der Kommunikation. Zunächst erfolgt die Festlegung der Zielinhalte für jede Zielgruppe und die Vereinheitlichung in einem Konzept. Die Definition der kommunikativen Leitidee dient zum Umsetzen der strategischen Positionierung in den Botschaften und Grundaussagen des Unternehmens. Analog zur Leitidee werden innerhalb der Kommunikationsinstrumente Leitinstrumente spezifiziert, die ausschlaggebend für die gewünschte Positionierung ausschlaggebend sind (Bruhn, 2003, 164f). Zudem wird die Kommunikation klar geregelt (Bruhn, 2003, 190ff, 341f).

In der sechsten Stufe erfolgt die Festlegung und Verteilung des Kommunikationsbudgets. Die Aufteilung des Gesamtbudgets wird von Bruhn als Managementaufgabe klassifiziert. In dieser Stufe endet der Planungsprozess und es erfolgt der Einstieg in Stufe sieben und damit die Realisierung der Gesamtkommunikation. Bei der Realisierung liegt der Fokus auf der inhaltlichen, organisatorischen und personellen Integration, die nachfolgend noch eine kurze Betrachtung finden werden.

Der Planungsansatz endet mit der Erfolgskontrolle der Gesamtkommunikation und der Kommunikationsinstrumente und -mittel (vgl. Bruhn, 2003, 297ff). Die Integrierte Kommunikation stellt an die Organisation des Unternehmens eine besondere Herausforderung. Im Zentrum steht die organisatorische Integration der einzelnen Kommunikationsinstrumente und deren Koordination.

Da klassische Organisationsstrukturen den Anforderungen kaum gerecht werden (Bruhn, 2003, 201ff), schlägt er eine idealtypische organisatorische Gestaltung vor, die Projektorganisation mit interdisziplinären Teams und Lenkungsgremium vorsieht (Bruhn, 2003, 268f). Der Vorteil liegt in der konsequenten Einhaltung zeitlicher Dimensionen, das Aufheben von Hierarchie- und Ressortdenken sowie die Orientierung auf das Ergebnis.

Mit der strukturellen Einbindung der integrierten Kommunikation in ein Unternehmen sind längst noch nicht alle Schritte getan. Das Bereichs- und 
Abteilungsdenken gilt als Barriere (vgl. Bruhn \& Boenigk, 1999, 78ff sowie Duncan \& Moriarty, 1997, 29f). Die Lösung zum Abbau der Barrieren obliegt dem Personalmanagement im Unternehmen, das durch geeignete Maßnahmen der Personalbestandsanalyse, -bedarfsanalyse, -beschaffung, -entwicklung, -freisetzung, -führung und des Personaleinsatzes die Integrierte Kommunikation zu fördern hat.

Prinzipiell kann die Umsetzung der integrierten Kommunikation nur erfolgreich durchgeführt werden, wenn im Unternehmen ein Integrationsbewusstsein geschaffen wird. Bruhn schlägt dafür einen Kommunikationsmanager als Leiter des beschriebenen Lenkungsgremiums innerhalb der Projektorganisation vor. Ihm obliegen dabei sowohl Weisungsbefugnisse als auch Entscheidungsbefugnisse (vgl. Bruhn, 2003, 321f).

Dabei hat der Manager mehrere Funktionen auszuüben: Die Planungsund Kontrollfunktion, die Initiierungsfunktion, die Beratungsfunktion, die Informationsfunktion sowie die Entscheidungsfunktion (vgl. Bruhn, 2009, 269ff).

Für Bruhn stellt die interne Kommunikation ein zentrales strategisches Instrument dar (vgl. Bruhn, 2003, 351f), welches die Ziele verfolgt, die Mitarbeiter früher und umfassender zu informieren als externe Zielgruppen und damit die Motivation der Mitarbeiter zu erhöhen, eine Identifikation mit dem Unternehmen zu schaffen sowie glaubwürdige Meinungsmultiplikatoren aus den Mitarbeitern zu bilden.

Für Bruhn zählt zum Modell der integrierten Kommunikation ein umfassendes Kommunikationscontrolling. Er sieht im Controlling eine Sicherstellung der Effektivität und Effizienz der integrierten Kommunikation (Bruhn, 2009, 361). Konkret soll sichergestellt werden, ob die gesetzten Ziele erreicht wurden, welche Maßnahmen am meisten Erfolg hatten.

Bruhns Modell weist eine betriebswirtschaftliche Sicht der Unternehmenskommunikation auf. Die Notwendigkeit der Integrierten Kommunikation liegt für Bruhn in zwei Aspekten: zum einen stellt auch er ein überhöhtes Kommunikationsaufkommen fest, zum anderen sieht er in der Vielzahl an teilweise neuen Kommunikationskanälen und -instrumenten eine notwendige Integration in das bestehende Portfolio (vgl. Bruhn, 1995, 37). Positiv hervorzuheben ist, dass die strategische und taktische Planung in Bruhns Konzept berücksichtigt werden - es gibt konkrete Aussagen zu Botschaften, Zeitplänen, Richtlinien und organisatorischer Verantwortung. Da die Markenstrategie Ausgangspunkt für die Kommunikationsstrategie ist, gehen Unternehmensstrategie und Außendarstellung einher. Bruhns Konzept vernachlässigt das klassische Marketing, er sieht darin kein Beziehungsmarketing, sondern eine Instrumentenbox. Die Instrumente werden nach dem Einfluss aufeinander hierarchisiert und durch Bruhns Konzept aufeinander abgestimmt und optimiert. 


\section{Modell nach Gronstedt}

Das Konzept von Gronstedt basiert auf einem Stakeholder-Relations-Modell. Mit der Begründung, dass Kunden diejenige Bezugsgruppe darstellen, die das Erreichen der Organisationsziele am meisten beeinflussen, stellt er sie ins Zentrum seiner Überlegungen. In Gronstedts Modell sind alle anderen Bezugsgruppen mit der zentralen Bezugsgruppe der Kunden verbunden. Deshalb wird bei Gronstedt jede Bezugsgruppenkommunikation als integraler Teil der Kommunikation mit den Kunden behandelt. Zweck der Kommunikation ist auch hier der Aufbau langwährender Beziehungen zwischen Unternehmen und Kunden bzw. anderen Bezugsgruppen, welche alle mit den Kunden in Verbindung stehen (vgl. Gronstedt, 1996, 292).

Im Vordergrund steht für ihn die Integration von Marketingkommunikation und Public Relations, da er eine Überschneidung von Märkten und Teilöffentlichkeiten, ebensowieeineÜberschneidunginderVerwendungderKommunikationsinstrumente sowohl im der Marketingkommunikation als auch in der Öffentlichkeitsarbeit wahrgenommen hat (vgl. Gronstedt, 1996, 289ff). Gronstedt lehnt in diesem Zusammenhang die Trennung nach Kommunikationsverantwortlichen ab (vgl. Gronstedt, 1996, 292), sondern schlägt ein Modell vor, in dem alle Bezugsgruppen mit Kommunikationsinstrumenten angesprochen werden, welche wiederum in empfangbare, interaktive und Sendeinstrumente kategorisiert werden. Im Zentrum des Modells steht der Kunde, dessen gute Beziehung zum Unternehmen die essentielle Voraussetzung für den Erfolg ist. Alle anderen Anspruchsgruppen unterliegen einer Dynamik und können von Zeit zu Zeit wechseln, jedoch sind alle Bezugsgruppen mit dem Kunden verbunden. Folglich wird jede Handlung in der Bezugsgruppe Auswirkungen auf die Beziehung zum Kunden haben. Als möglicher Ansatz, den optimalen Mix an Kommunikationsinstrumenten zu finden, um die Bezugsgruppen zu erreichen, dient eine Datenbank, die alle Marketing und PRInstrumente organisiert. Nach der Kategorisierung müssen die Sendeinstrumente nach einheitlichen Botschaften und Bildern, nach gemeinsamen kreativen Elementen und zeitlich abgestimmt werden. Grundsätzliches Ziel der Aufteilung der Kommunikationsinstrumente in die drei Kategorien ist der Aufbau einer langfristigen Beziehung zu den Zielgruppen.

Das Konzept von Gronstedt untersucht vor allem die Stakeholder und deren Konstellationen. In die Betrachtung fließen die spezifischen Wünsche und Bedürfnisse von Stakeholdern ein, ebenso deren Erfassung in Gruppen. Dabei beschreibt Gronstedt eine Auflösung der Grenzen zwischen Kommunikation, Marketing und PR, da die Stakeholdergruppen sich überlappen und miteinander interagieren. Da Gronstedt einen Ansatz wählt, der auf dem Beziehungsmarketing basiert, fehlt in seiner Betrachtung der klassische Unternehmensbezug. Für ihn ist relevant, dass die Bezugsgruppen mit dem Unternehmen vernetzt werden. In seinem Gesamtkonzept bleibt Gronstedt bei seinem Stakeholderrelations-Ansatz, der viele 
Bezugsgruppen und Beziehungen definiert, bei der integrativen und strukturellen Ausführungen eher vage bleibt.

\section{Modell nach Zerfaß}

Das Modell der integrierten Kommunikation basiert bei Zerfaß auf einem organisationstheoretischen Ansatz. Er versteht unter dem Begriff der Unternehmenskommunikation im weitesten Sinne alle Kommunikationshandlungen von und in erwerbswirtschaftlichen Organisationen (vgl. Steinmann \& Zerfaß, 1995, 18).

Im Zentrum seines Modells steht die Unternehmensstrategie an der sich alle Kommunikationsinstrumente ausrichten. Gleichzeitig übernimmt die interne und externe Unternehmenskommunikation eine entscheidende strategische Rolle im Rahmen des strategischen Managements. Zerfaß unterteilt die Unternehmenskommunikation in die Bereiche Organisationskommunikation, Marktkommunikation und Public Relations (vgl. Zerfaß, 1996, 289ff).

Die Integrierte Kommunikation hat nach Zerfaß nur Erfolg, wenn die in den Einzeldisziplinen abgestimmten Kommunikationsaktivitäten einen optimalen Beitrag zum Unternehmenserfolg leisten. Der Autor identifiziert zwei Ansatzpunkte für die Integration: die Kommunikationshandlung, also die inhaltliche, formale und zeitliche Integration und den Bereich des Kommunikationsmanagements. Zerfaß sieht analog zu Bruhn die Notwendigkeit einer kommunikativen Leitidee, identifiziert darüber hinaus jedoch die Notwendigkeit eines zusätzlichen Steuerungskonzepts, das über die inhaltliche Planung hinausgeht. Das Management nimmt in seiner Betrachtung eine zentrale Rolle ein, die er um die Aufgaben Planung und Kontrolle der integrierten Kommunikation, sowie die Organisation, das Personalmanagement und die Mitarbeiterführung erweitert. (vgl. Steinmann \& Zerfaß, 1995, 38ff). Unter Planung versteht Zerfaß die Definition von Kernbotschaften und Gestaltungsprinzipien. Der Autor rät allerdings von einer Allmacht in der Planung durch das Management ab (vgl. Steinmann \& Zerfaß, 1995, 38), da zukünftige Entwicklungen nicht durch wenige Planer abzusehen sind. Stattdessen sind Integrationsaufgaben nicht allein Aufgabe eines zentralen Planers, sondern alle Managementfunktionen wie die Organisation oder das Personalmanagement müssen in die Planung integriert werden. Neben der Topdown-Planung kommt dabei der Bottom-up-Planung eine tragende Rolle zu, da sich konkrete Kommunikationsprobleme nicht zentral vorhersagen lassen.

Eine große Bedeutung misst Zerfaß auch der Kontrolle bei, die nicht allein in einem Soll-Ist-Vergleich verankert sein kann, sondern folgende drei Ebenen umschließt: Die strategische Überwachung dient als globale Kernfunktion. Daneben müssen gesetzte Annahmen kontrolliert (Prämissenkontrolle) und während der Durchführung die relevanten Informationen eruiert werden (Durchführungskontrolle) (vgl. Steinmann \& Schreyögg, 2005, 282ff). 
Für die Schaffung geeigneter Strukturen und Organisationseinheiten für die Integrierte Kommunikation schlägt Zerfaß dezentrale und multipersonale Strukturen (Projektgruppen) vor, die durch eine Koordinationsstelle in der Funktion eines „Coachings“ ergänzt werden. Grundsätzlich stellt er fest, dass der Integrationsgedanke in der Unternehmenskultur verankert sein muss, um langfristig erfolgreich zu sein.

Zerfaß setzt bei einer breiten gesellschaftsorientierten Unternehmensperspektive an, die den Rahmen für seine Unternehmenskommunikation bildet. Für ihn ist die integrierte Unternehmenskommunikation ein zentrales Steuerungselement. Interessant ist, dass Zerfaß betont, dass Integration nicht Einheitlichkeit bedeutet, da unterschiedliche Probleme angepasste Lösungen brauchen. Die Konzepte der Marke, Markenpositionierung und Beziehungsmarketing spielen bei Zerfaß keine herausragende Rolle, weshalb sein Ansatz eher organisationstheoretisch angelegt ist. Die Einbindung von interner und externer Kommunikation sowie der an der Kommunikation beteiligter Unternehmen in das Konzept der integrierten Kommunikation sind positiv zu würdigen.

\section{Zusammenfassung und Fazit \\ der Modelle integrierter Kommunikation}

Integrierte Unternehmenskommunikation ist ein Planungs-, Organisations- und Optimierungsprozess (vgl. Kirchner, 2001, 173), der in verschiedenen Modellen beschrieben wurde, die sich teilweise deutlich unterscheiden. Während das Modell von Thorson und Moore sehr stark auf den Kunden orientiert ist, wagt Gronstedt einen Blick auf größer gefasste Zielgruppen. Sie eint aber gleichfalls die Unklarheit, welche Praxistauglichkeit und -relevanz die Modelle aufweisen. Die Konzepte von Bruhn und Zerfaß gehen von einem strategischen Prozess ausgehend von der Unternehmenspositionierung und -strategie aus. Sie unterscheiden sich aber in ihrer Zielsetzung. Alle Modelle haben Stärken und Schwächen und haben jeweils einen stärkeren Theorie- oder Praxisbezug.

Insbesondere durch die ausführliche Darstellung des sowohl strategischen als auch operativen Planungsprozesses und der organisatorischen sowie personellen Gestaltung im Unternehmen stellt Manfred Bruhn einen Ansatz vor, der eine hohe praktische Relevanz für Unternehmen hat und ein marketinggeprägtes Verständnis verdeutlicht. Sein Modell der integrierten Kommunikation zeichnet sich vor allem durch den sehr präzisen und ausführlichen Planungsprozess für die Umsetzung und durchseineTiefeunddenPraxisbezugaus.EswirdeinpersuasiverKommunikationsstil deutlich, mit dessen Hilfe die Zielgruppen von den ausgesendeten Botschaften des Unternehmens überzeugt werden sollen. Hervorzuheben ist die klare Struktur, der schlüssige Aufbau und der hohe Konkretisierungsgrad des Bruhnschen Ansatzes. Die einzusetzenden Kommunikationsinstrumente werden differenziert beschrieben 
und ihre Beziehungen im Detail analysiert. Dadurch werden auch relevante organisatorische und personelle Gesichtspunkte berücksichtigt und die Kunden als wichtige Zielgruppe aufgeführt. Prägendes Element des Ansatzes bilden die detaillierten Ausführungen zu den einzelnen Aufgaben innerhalb des dargestellten Managementprozesses. Der Wissenschaftler erarbeitet ein hierarchisches System, in dem Ziele, Aussagen und Kommunikationsinstrumente in inhaltlicher, formaler und zeitlicher Hinsicht aufeinander abgestimmt werden.

Jedes der Modelle hat durch den jeweiligen Ansatz auch das Potential zur Kritik. Kirchner schätzt ein, dass die ausgewogensten Modelle von Bruhn und Zerfaß stammen (vgl. Kirchner, 2001, 174), wobei das Modell von Zerfaß aufgrund seiner sehr theoretischen Betrachtung vor allem einer gewissen theoretischen Diskussion unterliegt.

Um auf die Eingangsfragen zurückzukommen lässt sich abschließend festhalten, dass die integrierte Kommunikation sowohl ein theoretisches Konstrukt ist, das modellhaft idealtypische Zustände untersucht und gleichzeitig relevante Randbedingungen unscharf betrachtet aber ebenso gut insbesondere im Fall von Bruhn eine große Praxisrelevanz aufweist. Das Konzept von Bruhn bietet durch dessen Detaillierungsgrad ausreichend Anknüpfungs- und Vergleichspunkte für Unternehmen. Die integrierte Kommunikation in Gänze ist damit sowohl theoretisches Konstrukt als auch praktische Handlungsempfehlung.

\section{Praxisanwendung der integrierten Kommunikation}

Die zuvor getroffene Einschätzung zum Modell der integrierten Kommunikation nach Manfred Bruhn hat sich auch in der Praxis bestätigt. Bruhn selbst hat sein Modell in mehreren Studien in Unternehmen untersucht. Bereits 2005 ist er zur Einschätzung gelangt, dass rund 65 Prozent der befragten Unternehmen sich mit integrierter Kommunikation beschäftigen (vgl. Bruhn, 2009, 41). Im Jahr 2014 hat er seine Studie wiederholt und insgesamt 138 Unternehmen zum Entwicklungsstand der integrierten Kommunikation in den deutschsprachigen Ländern befragt. Die Ergebnisse beider Studien hat er miteinander ins Verhältnis gesetzt und dabei nachgewiesen, dass die Relevanz der integrierten Kommunikation in der Praxis existiert (vgl. Bruhn, 2014, 2). Diese Behauptung stützt sich insbesondere auf die detaillierten Aussagen aus der Auswertung, in der er einzelne Faktoren und Elemente seines integrierten Kommunikationskonzepts erfragt hat. Aus dieser Auswertung lässt sich nicht allein schlussfolgern, dass das Konzept in der Praxis angewendet wird, sondern auch, welche Faktoren des Konzepts in der Praxis tatsächlich von großer Bedeutung sind.

So geben insgesamt 36,2 Prozent der Unternehmen an, dass das Konzept der integrierten Kommunikation auf der Unternehmensphilosophie aufbaut. Da die Negation lediglich 7,2 Prozent der Befragten betrifft, ist zumindest eine Zustimmung 
der Unternehmen zu verzeichnen, dass die integrierte Kommunikation auf Basis der Philosophie des Unternehmens zu verankern ist (vgl. Bruhn, 2014, 38f). Den für die integrierte Kommunikation wichtigen Merkmalen der inhaltlichen und formalen Abstimmung wird grundsätzlich Rechnung getragen. Allerdings geben die Unternehmen an, dass die inhaltliche Abstimmung der Instrumente des Marketingmixes und die Vorgabe der Kommunikationsbotschaften Schwierigkeiten bereiten, während die rein formale Abstimmung gut gelingt (vgl. Bruhn, 2014, 38).

Bei der Interpretation der Ergebnisse fällt auf, dass die befragten Unternehmen auf die Frage: „Welche der folgenden Merkmale weist das Konzept der Integrierten Kommunikation in Ihrem Unternehmen auf?" (Bruhn et al. 2014: 139) folgende Elemente in ihrem Unternehmen in Bezug auf die integrierte Kommunikation als wichtig einschätzen (vgl. Bruhn, 2014, 38):

- Festgeschriebene Kommunikationsziele.

- Strategische Positionierung für die Kommunikation.

- Festlegung der Zielgruppen.

- Abstimmung mit den allgemeinen Unternehmenszielen.

- Vorschriften in Bildsprache und Corporate Design.

- Unterstützung durch die Führungsebene.

- Verbindlich für alle Kommunikationsbeteiligten.

Diese Merkmale scheinen in der Praxis für ein Gelingen der integrierten Kommunikation nach Bruhn in besonderem Maß erforderlich zu sein.

Bruhn hat deshalb im Rahmen seiner Analyse explizit die Zusammenhänge zwischen Konzeptmerkmalen und der Erfolgswirkung der integrierten Kommunikation untersucht. Für ihn haben sich eine Vielzahl an erfolgswirksamen Konzeptmerkmalen ergeben.

So gilt die Vorgabe der Kommunikationsbotschaften in Abstimmung mit den allgemeinen Unternehmenszielen als relevanter Beitrag zur Steigerung der Bekanntheit. Gleiches gilt auch für den Fall, dass das Konzept der integrierten Kommunikation auf der Unternehmensphilosophie basiert und mit den übrigen Instrumenten des Kommunikationsmixes abgestimmt wird. Dann ergibt sich sogar ein positiver Effekt auf das Image. Ist das Konzept mit der Unternehmensphilosophie abgestimmt und die Zielgruppen für die Kommunikation festgelegt, ist die Kundenzufriedenheit hoch.

Positiv auf die Kundenbindung wirkt sich zudem aus, wenn die Führungsebene das Konzept der integrierten Kommunikation unterstützt. Ein positiver Effekt auf Umsatz und Gewinn ist zu verzeichnen, wenn neben der Abstimmung mit der Unternehmensphilosophie und den Unternehmenszielen auch Kommunikationsbotschaften vorgegeben werden (vgl. Bruhn, 2014, 42).

Im Kern gelten bei den Unternehmen die Abstimmung auf die Unternehmensphilosophie und die Unternehmensziele, eine Festlegung von Kommunikationsbotschaften, Zielgruppen und Instrumenten und eine Unterstützung durch die 
Führungsebenen als Erfolgskriterien für die integrierte Kommunikation. Dies kann ergänzt werden durch die im Verlauf der Studie dargestellte Betrachtung der Strukturen. Als Erfolg sehen die Unternehmen an, wenn eine regelmäßige Zusammenarbeit zwischen den Kommunikationsabteilungen erfolgt, die sich auch in einem gegenseitigen Informationsaustauschsystem niederschlägt (vgl. Bruhn, 2014, 44f.). Außerdem scheint der Studie nach die Implementierung integrationsorientierter Prozesse erfolgsversprechend $\mathrm{zu}$ sein, so dass eine abteilungsübergreifende Abstimmung hinsichtlich der Kommunikation sichergestellt wird (vgl. Bruhn, 2014, 56f.).

Die Studien zeigen zweierlei Ergebnisse. Erstens scheint das Modell von Bruhn auch in der Praxis anwendbar zu sein. Die theoriegeleitete Analyse und die Faktoren lassen sich nach Bruhn auch in der Praxis wiederfinden und nachweisen. Zweitens gilt jedoch auch, dass die Theorie der integrierten Kommunikation nach Bruhn nicht priorisiert, die Praxis dagegen schon. Einige Punkte, die in der Theorie gleichrangig betrachtet werden, sind in der Praxis noch unterentwickelt oder spielen keine herausragende Rolle, beispielsweise die zeitliche Integration. Im Hinblick auf eine weiterführende Untersuchung der integrierten Kommunikation nach Bruhn in der Praxis, muss das theoretische Konzept hierarchisiert werden. Nur so lassen sich aus dem theoretischen Konzept praktische Handlungsanweisungen ableiten, auch wenn die Praxisanwendung durch die Untersuchungen bereits nachgewiesen werden konnte. Die Hierarchisierung und Priorisierung der einzelnen Elemente der integrierten Kommunikation muss Teil weiterer Forschungsvorhaben sein.

\section{Literaturverzeichnis}

1. Bruhn, M. (1992). Integrierte Unternehmenskommunikation: Ansatzpunkte für eine strategische und operative Umsetzung integrierter Kommunikationsarbeit. Stuttgart: Schäffer-Poeschel.

2. Bruhn, M. (1995). Integrierte Unternehmenskommunikation. 2., überarbeitete und erweiterte Aufl. Stuttgart: Schäffer-Poeschel Verlag.

3. Bruhn, M. (2006). Integrierte Kommunikation in den deutschsprachigen Ländern: Bestandsaufnahme in Deutschland, Österreich und der Schweiz. Wiesbaden: Gabler.

4. Bruhn, M. (2009). Integrierte Unternehmens- und Markenkommunikation. Strategische Planung und operative Umsetzung. 5., überarb. und aktualisierte Aufl. Stuttgart: SchäfferPoeschel.

5. Bruhn, M. (2014). Integrierte Unternehmens- und Markenkommunikation: Strategische Planung und operative Umsetzung (6. Aufl.). Stuttgart: Schäffer-Poeschel.

6. Bruhn, M. (2016). Relationship Marketing: Das Management von Kundenbeziehungen. Vahlen.

7. Bruhn, M., Boenigk, M. (1999). Integrierte Kommunikation: Entwicklungsstand in Unternehmen. Wiesbaden: Gabler. 
8. Bruhn, M., Martin, S., Schnebelen, S. (2014). Integrierte Kommunikation in der Praxis. Entwicklungsstand in deutschsprachigen Unternehmen. Wiesbaden: Springer Gabler (Basler Schriften zum Marketing, 32). Online verfügbar unter http://dx.doi. org/10.1007/978-3-658-05464-9.

9. Bruhn, M., Esch, F.-R., Langner, T. (Hrsg.) (2016). Handbuch Instrumente der Kommunikation (2. Aufl.). Wiesbaden: Springer Gabler.

10. Bruhn, M., Zimmermann, A. (1993). Integrierte Kommunikationsarbeit in deutschen Unternehmen: Ergebnisse einer Unternehmensbefragung. In M. Bruhn \& H. D. Dahlhoff (Hrsg.), Effizientes Kommunikationsmanagement (pp. 145-215). Stuttgart: SchäfferPoeschel.

11. Brünne, M., Esch, F.R., Ruge, H.-D. (1987). Berechnung der Informationsüberlastung in der Bundesrepublik Deutschland, Bericht des Instituts für Konsum- und Verhaltensforschung der Universität des Saarlandes, Saarbrücken.

12. Duncan, T. R., Moriarty, S. E. (1997). Driving brand value using integrated marketing to manage profitable stakeholder relationships. New York: McGraw-Hill.

13. Duncan, T. R., Moriarty, S. E. (1998). A Communication-Based Marketing Model for Managing Relationships. Journal of Marketing, 62(2), 1-13.

14. Eagle, L., Kitchen, P., Bulmer, S. (2007). Insights into interpreting integrated marketing communications. European fournal of Marketing, 41.

15. Esch, F. R. (1998). Wirkung integrierter Kommunikation: Ein verhaltenswissenschaftlicher Ansatz für die Werbung. Wiesbaden: DUV.

16. Esch, F. R. (2001). Wirksame Markenkommunikation bei steigender Informationsüberlastung der Konsumenten. In R. Köhler (Hrsg.), Erfolgsfaktor Marke: neue Strategien des Markenmanagements. (pp. 71-89) München: Verlag Vahlen, S.

17. Esch, F. R. (2011). Wirkung integrierter Kommunikation: Ein verhaltenswissenschaftlicher Ansatz für die Werbung (5. Aufl.). Wiesbaden: Gabler.

18. Gronstedt, A. (1996). Integrated Communications at America's Leading Total Quality Management Corporations. Public Relations Review, 22(1).

19. Gronstedt, A. (2000). The customer century: lessons from world-class companies in integrated marketing and communications. New York; London: Routledge.Kappler, Rehkugel 1991, $942 f$.

20. Kerr, G., Patti, C., Chien, M. (2008). Integrated Marketing Communications (IMC): new discipline with an old learning approach: A Syllabi Analysis. Online im Internet: http://130.195.95.71:8081/WWW/ANZMAC2004/CDsite/papers/Kerr1.PDF [Stand 14. September 2018].

21. Kirchner, K. (2001). Integrierte Unternehmenskommunikation. Theoretische und empirische Bestandsaufnahme und eine Analyse amerikanischer Großunternehmen. Wiesbaden: Westdeutscher Verlag.

22. Kitchen, P. J., Pelsmacker, P. (2004). Integrated marketing communications. A primer. London, New York: Routledge. Online verfügbar unter http://site.ebrary.com/lib/ alltitles/docDetail.action?docID=10093635. 
23. Kroeber-Riel, W. (1993). Bildkommunikation. Imagerystrategien für die Werbung. München.

24. Kroeber-Riel, W., Esch, F.-R. (2004). Strategie und Technik der Werbung, Verhaltenswissenschaftliche Ansätze, 6. Auflage, Verlag W. Kohlhammer, Stuttgart.

25. Maletzke, G. (1998). Kommunikationswissenschaft im Überblick. Grundlagen, Probleme, Perspektiven. Opladen/Wiesbaden.

26. Merten, K. (1994). Wirkungen von Kommunikation. In Merten, K.; Schmidt, S. J.; Weischenberg, S. (Hrsg.), Die Wirklichkeit der Medien. Eine Einführung in die Kommunikationswissenschaft (pp. 291-328). Opladen.

27. Moore, J., Thorson, E. (1996). Strategic Planning for Integrated Marketing Communication Programms. An Approach to Moving from Chaotic Toward Systematic. In E. Thorson, J. Moore (Eds.), Integrated Communications: synergy of Persuasive Voices (pp.135-152). Mahwah; New Jersey: Lawrence Erlbaum Associates.

28. Pelsmacker, P., Geuens, M.; van den Bergh, J. (2017). Marketing communications. A European perspective. 6th edition. Harlow: Pearson Education.

29. Pepels, W. (1995). Käuferverhalten und Marktforschung: Eine praxisorientierte Einführung.

30. Schultz, D. E.; Kitchen, P. (1992). Integrated Marketing Communications in US Advertising Agencies: An Exploratory Study. Journal of Advertising Research, 37(5), 1997, 7-17

31. Schultz, D. E.; Kitchen, P. J. (2000). Communicating Globally - An Integrated Marketing Approach. Lincolnwood: NTC Business Books.

32. Schultz, D. E.; Schultz, H. F. (1998). Transitioning marketing communication into the twenty-first century. Journal of Marketing Communication, 4(1).

33. Schultz, D. E., Schultz, H. F. (2004). IMC: The next generation. New York, NY: McGrawHill.

34. Schultz, D. E., Tannenbaum, S. I., Lauterborn, R. F. (1993). Integrated Marketing Communications: Putting it together \& making it work by. 1. Auflage, Lincolnwood

35. Seiffert, H., Radnitzky, G. (1994). Handlexikon zur Wissenschaftstheorie. DTV, München.

36. Steinmann, H., Schreyögg, G. (2005). Management: Grundlagen der Unternehmensführung - Konzepte - Funktionen - Fallstudien. 6., vollständig überarbeitete Aufl., Wiesbaden: Verlag Gabler.

37. Steinmann, H., Zerfaß, A. (1995). Management der integrierten Unternehmenskommunikation: Konzeptionelle Grundlagen und strategische Implikationen. In R. Ahrens, H. Scherer \& A. Zerfaß (Hrsg.), Integriertes Kommunikationsmanagement (pp. 11-50). Frankfurt a. M.: F.A.Z-Institut.

38. Stumpf, M. (2005). Erfolgskontrolle der Integrierten Kommunikation: Messung des Entwicklungsstandes integrierter Kommunikation in Unternehmen. Wiesbaden: Verlag Gabler.

39. Zerfaß, A. (1996). Unternehmensführung und Öffentlichkeitsarbeit: Grundlegung einer Theorie der Unternehmenskommunikation und Public Relations. Opladen: Westdeutscher Verlag.

40. Zerfaß, A. (2010). Unternehmensführung und Öffentlichkeitsarbeit: Grundlegung einer Theorie der Unternehmenskommunikation und Public Relations (3. Aufl.). Wiesbaden: Springer VS. 
41. Zerfaß, A. (2014). Unternehmensführung und Kommunikationsmanagement: Strategie, Management und Controlling. In A. Zerfaß \& M. Piwinger (Hrsg.), Handbuch Unternehmenskommunikation, 2. Aufl., (pp. 21-79). Wiesbaden: Springer Gabler.

42. Zerfaß, A., Borchers, N. S. (2017). Integrierte Kommunikation 2017 - Studie zum Status Quo und Verständnis von Integrierter Kommunikation in Deutschland Frankfurt am Main. Leipzig: F.A.Z.-Institut, Universität Leipzig.

43. Zerfaß, A., Piwinger, M. (Hrsg.) (2014). Handbuch Unternehmenskommunikation (2. Aufl.). Wiesbaden: Springer Gabler. 